\title{
Anomalous fermion number non-conservation on the lattice
}

\author{
István Montvay* \\ CERN, Geneva, Switzerland
}

\begin{abstract}
The anomaly for the fermion number current is calculated on the lattice in a simple prototype model with an even number of fermion doublets.
\end{abstract}

\section{INTRODUCTION}

Fermion number, which is the sum of baryon number and lepton number $(B+L)$, is not conserved in the Standard Model [1]. This is due to the anomaly in the fermion current. Under "normal" conditions there is, however, a strong suppression factor

$$
\exp \left(-4 \pi / \alpha_{W}\right) \simeq 10^{-150}
$$

which makes $(B+L)$ violation unobservable. At high temperature and/or high fermion densities (at high energies) the non-conservation is amplified. This may explain the small baryon asymmetry of the universe, which could arise via this mechanism at the cosmological electroweak phase transition. (For references to the extensive literature on this subject, see the reviews in ref. [2].)

The lattice formulation of the anomalous fermion number non-conservation is problematic [3], because it has to do with the chiral $\mathrm{SU}(2)_{\mathrm{L}}$ gauge coupling and, as is well known, there is a difficulty with chiral gauge fields on the lattice (see, for instance, the review [4). There is, however, an approximation of the electroweak sector of the Standard Model, which can be studied with standard lattice techniques, namely the limit when the $\mathrm{SU}(3)_{\text {colour }} \otimes \mathrm{U}(1)_{\text {hypercharge }}$ gauge couplings are neglected. The usefulness of this limit for lattice studies was particularly emphasized in earlier works by Lee and Shrock (see [5] and references therein). In their phase structure studies staggered fermions were used. Here Wilson fermions will be considered, which naturally lead to the mirror fermion action for chiral gauge theories [6]. There is now a growing amount of experience with this action, without $\mathrm{SU}(2)_{\mathrm{L}}$ gauge field, in the numerical simulation studies of the allowed region of renormalized quartic and Yukawa couplings [7, 8, 9]. The inclusion of the $\mathrm{SU}(2)_{\mathrm{L}}$ gauge field in the simulation algorithms is straightforward;

*Permanent address: DESY, Hamburg, FRG 
therefore one can start to speculate about the possibility to explore some features of the violation of the fermion number conservation. In order to understand the mechanism of fermion number non-conservation on the lattice, let us see how the relevant anomalous WardTakahashi identity arises in this formulation.

\section{LATTICE ACTION}

Let us consider a simple prototype model, which is the extension of the standard $\mathrm{SU}(2)_{\mathrm{L}}$ Higgs model by an even number $2 N_{f}$ of fermion doublets. In the Standard Model we have $N_{f}=6$ (for simplicity, we consider Dirac neutrinos, but the massless neutral right-handed neutrinos decouple [10]). In what follows we take, for simplicity, $N_{f}=1$, but the extension to $N_{f}>1$ is trivial. The lattice action depends on the matrix scalar field $\varphi_{x}=\phi_{0 x}+i \phi_{s x} \tau_{s}$ (with four real fields $\phi_{S=0, \ldots, 3}$ ) and the fermion doublet fields $\psi_{(1,2) x}$ :

$$
S=S_{\text {scalar }}+S_{\text {fermion }}
$$

The standard Higgs-model action is

$$
\begin{gathered}
S_{\text {scalar }}=\frac{1}{4} \sum_{x}\left\{m_{0}^{2} \operatorname{Tr}\left(\varphi_{x}^{\dagger} \varphi_{x}\right)+\lambda\left[\operatorname{Tr}\left(\varphi_{x}^{\dagger} \varphi_{x}\right)\right]^{2}\right. \\
\left.+\sum_{\mu= \pm 1}^{ \pm 4}\left[\operatorname{Tr}\left(\varphi_{x}^{\dagger} \varphi_{x}\right)-\operatorname{Tr}\left(\varphi_{x+\hat{\mu}}^{\dagger} U_{x \mu} \varphi_{x}\right)\right]\right\} .
\end{gathered}
$$

The fermionic part contains the chiral gauge fields (with $U_{x \mu} \in \mathrm{SU}(2)$ and $P_{L, R}=\left(1 \mp \gamma_{5}\right) / 2$ )

$$
U_{(L, R) x \mu}=P_{(L, R)} U_{x \mu}+P_{(R, L)}
$$

and is given by

$$
\begin{gathered}
S_{\text {fermion }}=\sum_{x}\left\{\frac{\mu_{0}}{2}\left[\left(\psi_{2 x}^{T} \epsilon C \psi_{1 x}\right)-\left(\psi_{1 x}^{T} \epsilon C \psi_{2 x}\right)+\left(\bar{\psi}_{2 x} \epsilon C \bar{\psi}_{1 x}^{T}\right)-\left(\bar{\psi}_{1 x} \epsilon C \bar{\psi}_{2 x}^{T}\right)\right]\right. \\
-\frac{1}{2} \sum_{\mu}\left[\left(\bar{\psi}_{1 x+\hat{\mu}} \gamma_{\mu} U_{L x \mu} \psi_{1 x}\right)+\left(\bar{\psi}_{2 x+\hat{\mu}} \gamma_{\mu} U_{L x \mu} \psi_{2 x}\right)\right. \\
-\frac{r}{2}\left(\left(\psi_{2 x}^{T} \epsilon C \psi_{1 x}\right)-\left(\psi_{2 x+\hat{\mu}}^{T} \epsilon C U_{L x \mu} \psi_{1 x}\right)-\left(\psi_{1 x}^{T} \epsilon C \psi_{2 x}\right)+\left(\psi_{1 x+\hat{\mu}}^{T} \epsilon C U_{L x \mu} \psi_{2 x}\right)\right. \\
\left.\left.+\left(\bar{\psi}_{2 x} \epsilon C \bar{\psi}_{1 x}^{T}\right)-\left(\bar{\psi}_{2 x+\hat{\mu}} U_{R x \mu} \epsilon C \bar{\psi}_{1 x}^{T}\right)-\left(\bar{\psi}_{1 x} \epsilon C \bar{\psi}_{2 x}^{T}\right)+\left(\bar{\psi}_{1 x+\hat{\mu}} U_{R x \mu} \epsilon C \bar{\psi}_{2 x}^{T}\right)\right)\right] \\
\left.+\left(\bar{\psi}_{1 R x} G_{1} \varphi_{x}^{+} \psi_{1 L x}\right)+\left(\bar{\psi}_{1 L x} \varphi_{x} G_{1} \psi_{1 R x}\right)+\left(\bar{\psi}_{2 R x} G_{2} \varphi_{x}^{+} \psi_{2 L x}\right)+\left(\bar{\psi}_{2 L x} \varphi_{x} G_{2} \psi_{2 R x}\right)\right\} .
\end{gathered}
$$

Here $\epsilon=i \tau_{2}$ acts in isospin space, and $C$ is the fermion charge conjugation matrix. The Yukawa couplings $G_{1,2}$ can, in general, be arbitrary diagonal matrices in isospin space but, for simplicity, we shall here only consider the case with degenerate doublets $\left(G_{1,2}\right.$ proportional to the unit matrix). 
Instead of the off-diagonal Majorana mass $\mu_{0}$ and Majorana-like Wilson term (proportional to $r$ ), it is technically more convenient to consider a Dirac-like form with $\psi \equiv \psi_{1}$ and the mirror fermion field

$$
\chi_{x} \equiv \epsilon^{-1} C \bar{\psi}_{2 x}^{T}, \quad \bar{\chi}_{x} \equiv \psi_{2 x}^{T} \epsilon C .
$$

In terms of $\psi$ and $\chi$ one obtains the mirror fermion action for chiral gauge fields [6] ( $G_{\psi} \equiv$ $\left.G_{1}, G_{\chi} \equiv G_{2}\right)$ :

$$
\begin{gathered}
S_{\text {fermion }}=\sum_{x}\left\{\mu_{0}\left[\left(\bar{\chi}_{x} \psi_{x}\right)+\left(\bar{\psi}_{x} \chi_{x}\right)\right]-\frac{1}{2} \sum_{\mu= \pm 1}^{ \pm 4}\left[\left(\bar{\psi}_{x+\hat{\mu}} \gamma_{\mu} U_{L x \mu} \psi_{x}\right)+\left(\bar{\chi}_{x+\hat{\mu}} \gamma_{\mu} U_{R x \mu} \chi_{x}\right)\right.\right. \\
\left.\quad-r\left(\left(\bar{\chi}_{x} \psi_{x}\right)-\left(\bar{\chi}_{x+\hat{\mu}} U_{L x \mu} \psi_{x}\right)+\left(\bar{\psi}_{x} \chi_{x}\right)-\left(\bar{\psi}_{x+\hat{\mu}} U_{R x \mu} \chi_{x}\right)\right)\right] \\
\left.+\left(\bar{\psi}_{R x} G_{\psi} \varphi_{x}^{\dagger} \psi_{L x}\right)+\left(\bar{\psi}_{L x} \varphi_{x} G_{\psi} \psi_{R x}\right)+\left(\bar{\chi}_{L x} G_{\chi} \varphi_{x}^{\dagger} \chi_{R x}\right)+\left(\bar{\chi}_{R x} \varphi_{x} G_{\chi} \chi_{L x}\right)\right\} .
\end{gathered}
$$

This is the appropriate form of the fermion action in the phase with broken symmetry, as the investigations of the corresponding chiral Yukawa models show [11, 12, 7, 8, 9].

In the symmetric (i.e. confinement) phase, however, there is a natural alternative choice in terms of the reshuffled combinations [11]:

$$
\psi_{A x} \equiv \psi_{L x}+\chi_{R x}, \quad \psi_{B x} \equiv \chi_{L x}+\psi_{R x}
$$

On this basis the vector-like nature of the model becomes explicit $\left(\gamma_{5}\right.$ 's appear only in the Yukawa couplings). The SU(2) gauge field couples only to $\psi_{A}$, and the neutral doublet $\psi_{B}$ has only its Yukawa coupling.

Note the different rôles played by $\mu_{0}$ in the three lattice actions: in (5) it is an off-diagonal Majorana mass, in (7) the fermion-mirror-fermion mixing mass, whereas on the basis in (8) it is a common Dirac mass for $\psi_{A}$ and $\psi_{B}$. The physical interpretation of the model is, of course, given in terms of the action in (5).

Previous studies of the phase structure of the same continuum "target theory" in the staggered fermion formulation were usually done in a basis corresponding to (8), with the known differences between staggered and Wilson fermions (see, for instance, [13, 14, 5, 15]). In many cases the Yukawa couplings were omitted, and the $\psi_{B}$ field was not considered at all. Representing the fermion number anomaly both in terms of the fields in (5) and (8) is useful, because it gives the connection to the axial anomaly. This connection has recently been exploited also in ref. [16].

\section{THE ANOMALY}

On smooth background scalar and gauge fields $\left\{\varphi_{x}, U_{x \mu}\right\}$ the effective action is defined by

$$
\exp \left\{-S_{e f f}[U, \varphi]\right\} \equiv Z_{f 0}^{-1} \int[d \bar{\Psi} d \Psi] \exp \left\{-S_{f}[\Psi, \bar{\Psi}, U, \varphi]\right\}
$$

where $\Psi \equiv\{\psi, \chi\}$. An infinitesimal fermion number transformation is:

$$
\psi_{x}=\left(1+i \alpha_{x}\right) \psi_{x}^{\prime}, \quad \chi_{x}=\left(1-i \alpha_{x}\right) \chi_{x}^{\prime}
$$




$$
\bar{\psi}_{x}=\left(1-i \alpha_{x}\right) \bar{\psi}_{x}^{\prime}, \quad \bar{\chi}_{x}=\left(1+i \alpha_{x}\right) \bar{\chi}_{x}^{\prime} .
$$

This corresponds to the fact that the fermion number is defined to be +1 for the fields $\psi_{1,2}$ (and hence it is -1 for $\chi$ ).

The gauge-invariant fermion number current can be defined as

$$
J_{x \mu} \equiv \frac{1}{2}\left[\left(\bar{\psi}_{x+\hat{\mu}} \gamma_{\mu} U_{L x \mu} \psi_{x}\right)+\left(\bar{\psi}_{x} \gamma_{\mu} U_{L x \mu}^{\dagger} \psi_{x+\hat{\mu}}\right)-\left(\bar{\chi}_{x+\hat{\mu}} \gamma_{\mu} U_{R x \mu} \chi_{x}\right)-\left(\bar{\chi}_{x} \gamma_{\mu} U_{R x \mu}^{\dagger} \chi_{x+\hat{\mu}}\right)\right]
$$

Introducing the new integration variables $\left(\psi^{\prime}, \bar{\psi}^{\prime}, \chi^{\prime}, \bar{\chi}^{\prime}\right)$ in the path integral with action (可), one obtains with $\Delta_{\mu}^{b} f_{x} \equiv f_{x}-f_{x-\hat{\mu}}$ the lattice W-T identity

$$
\begin{gathered}
\left\langle\Delta_{\mu}^{b} J_{x \mu}\right\rangle_{f}=\left\langle 2 \mu_{0}\left[\left(\bar{\chi}_{x} \psi_{x}\right)-\left(\bar{\psi}_{x} \chi_{x}\right)\right]\right. \\
+\frac{r}{2} \sum_{\mu=1}^{4}\left[4\left(\bar{\chi}_{x} \psi_{x}\right)-\left(\bar{\chi}_{x+\hat{\mu}} U_{L x \mu} \psi_{x}\right)-\left(\bar{\chi}_{x} U_{L x \mu}^{\dagger} \psi_{x+\hat{\mu}}\right)\right. \\
-\left(\bar{\chi}_{x} U_{L x-\hat{\mu}, \mu} \psi_{x-\hat{\mu}}\right)-\left(\bar{\chi}_{x-\hat{\mu}} U_{L x-\hat{\mu}, \mu}^{\dagger} \psi_{x}\right)-4\left(\bar{\psi}_{x} \chi_{x}\right)+\left(\bar{\psi}_{x+\hat{\mu}} U_{R x \mu} \chi_{x}\right)+\left(\bar{\psi}_{x} U_{R x \mu}^{\dagger} \chi_{x+\hat{\mu}}\right) \\
\left.\left.+\left(\bar{\psi}_{x} U_{R x-\hat{\mu}, \mu} \chi_{x-\hat{\mu}}\right)+\left(\bar{\psi}_{x-\hat{\mu}} U_{R x-\hat{\mu}, \mu}^{\dagger} \chi_{x}\right)\right]\right\rangle_{f} .
\end{gathered}
$$

This has to be evaluated in the continuum limit, when the momenta of the external fields in lattice units are of the order $a(a \rightarrow 0)$.

For small lattice spacing $a$ the left-hand side of (12) is of order $a^{4}$ (note that for the moment we keep the bare parameters fixed, for instance, $\mu_{0}$ can be of order 1 ). Therefore diagrammatically the contributing graphs can have at most four external field legs. Explicit evaluation shows that in the present case only those with two or three external fields (i.e. the triangle and quadrangle graphs) contribute. Introducing the $\mathrm{SU}(2)$ field strength as usual by

$$
F_{\mu \nu}^{s}(x)=\partial_{\mu} A_{\nu}^{s}(x)-\partial_{\nu} A_{\mu}^{s}(x)+g \epsilon_{s t u} A_{\mu}^{t}(x) A_{\nu}^{u}(x),
$$

the result is (this time for $2 N_{f}$ fermion doublets)

$$
\left\langle\partial_{\mu} J_{\mu}(x)\right\rangle_{f}=\lim _{a \rightarrow 0}\left\langle\Delta_{\mu}^{b} J_{x \mu}\right\rangle_{f} a^{-4}=N_{f} g^{2} \epsilon_{\mu \nu \rho \sigma} F_{\mu \nu}^{s}(x) F_{\rho \sigma}^{s}(x) \mathcal{I}\left(r, \mu_{0}\right)
$$

Here the lattice integral $\mathcal{I}$ is given by

$$
\mathcal{I}\left(r, \mu_{0}\right) \equiv \frac{1}{(2 \pi)^{4}} \int_{-\pi}^{\pi} \frac{\mu_{k} \cos k_{1} \cos k_{2} \cos k_{3} \cos k_{4}}{\left(\bar{k}^{2}+\mu_{k}^{2}\right)^{3}} \cdot\left[r \sum_{\alpha=1}^{4} \bar{k}_{\alpha}^{2} / \cos k_{\alpha}-\mu_{k}\right] d^{4} k
$$

and the notations are

$$
\begin{gathered}
\mu_{k}=\mu_{0}+\frac{r}{2} \hat{k}^{2}, \\
\bar{k}_{\mu}=\sin k_{\mu}, \quad \hat{k}_{\mu}=2 \sin \frac{k_{\mu}}{2} .
\end{gathered}
$$

The integral $\mathcal{I}$ is the same as the one occurring in the chiral anomaly, and one can prove (see e.g. [17, 18])

$$
\mathcal{I}(r, 0)=\frac{1}{32 \pi^{2}} \quad \text { (independently from } r \text { ) }
$$


Note that in the present regularization scheme no other terms on the right-hand side of (14) occur. For instance, the scalar field having Yukawa couplings to the fermions does not contribute at all (although, of course, it appears on external legs of the graphs). This is different from the non-Abelian $\mathrm{U}(\mathrm{N}) \otimes \mathrm{U}(\mathrm{N})$ anomaly studied in ref. [19] in other regularization schemes (with different lattice actions), where the Bardeen-counterterms [20] are in general non-zero.

Equations (14) and (17) show that the correct continuum anomaly is reproduced at vanishing bare (Majorana) fermion mass $\mu_{0}=0$. It is, however, interesting to investigate the $\mu_{0}$ dependence of the lattice integral in (15). The numerical evaluation of the corresponding lattice sum $\mathcal{I}_{L}$ on $L^{4}$ lattices up to $L=200$ shows that $\mathcal{I}=\lim _{L \rightarrow \infty} \mathcal{I}_{L}$ is very small, probably $\mathcal{I}\left(\mu_{0}, r\right)=0$ for every positive $\mu_{0}$ [21]. This behaviour implies that the anomaly in (14) disappears at every positive $\mu_{0}$, and there is a singularity at $\mu_{0}=0$, where according to (17) the value of $\mathcal{I}$ is non-zero. The derivatives of $\mathcal{I}$ with respect to $\mu_{0}$ tend to infinity for $L \rightarrow \infty$; therefore we have, on the given external bosonic field configuration, for instance,

$$
\begin{aligned}
-\frac{\partial}{\partial \mu_{0}}\left\langle\Delta_{\mu}^{b} J_{x \mu}\right\rangle_{f} & =\left\langle\Delta_{\mu}^{b} J_{x \mu} \sum_{y}\left[\left(\bar{\chi}_{y} \psi_{y}\right)+\left(\bar{\psi}_{y} \chi_{y}\right)\right]\right\rangle_{f} \\
& -\left\langle\Delta_{\mu}^{b} J_{x \mu}\right\rangle_{f}\left\langle\sum_{y}\left[\left(\bar{\chi}_{y} \psi_{y}\right)+\left(\bar{\psi}_{y} \chi_{y}\right)\right]\right\rangle_{f} \rightarrow \infty .
\end{aligned}
$$

Here the infinity can be produced by the summation over $y$ because of the long-range correlation due to fermionic zero modes.

From the practical point of view the behaviour of $\mathcal{I}\left(r, \mu_{0}\right)$ implies that in numerical simulations one has to be careful in the extrapolation to $\mu_{0}=0$. The lattice volume should be small enough.

The functional dependence of the lattice integral $\mathcal{I}\left(r, \mu_{0}\right)$ on $\mu_{0}$ also illustrates how the anomaly is emerging from the explicit symmetry breaking present in the lattice action (2). In the case when the cut-off can be completely removed, this does not matter. Nevertheless, in theories with scalar fields there is the well-known "triviality problem", which implies that for finite renormalized couplings the lattice spacing cannot be taken to zero. This means that the unpleasant feature of the singular dependence of the anomaly on the bare fermion mass in principle remains. However, in order to understand the situation better, one has to consider the full theory with quantized bosonic fields, where a mixing of the renormalized composite operators has to be dealt with [22, 23].

Acknowledgements: It is a pleasure to thank L. Alvarez-Gaumé, A. Ringwald and M. Shaposhnikov for enlightening discussions, and the Theory Division of CERN for hospitality during the preparation of this proceedings contribution.

\section{References}

[1] G. 't Hooft, Phys. Rev. Lett. 37 (1976) 8; Phys. Rev. D14 (1976) 3432.

[2] M.E. Shaposhnikov, Nucl. Phys. B (Proc. Suppl.) 26 (1992) 78;

A. Ringwald, CERN preprint TH-6479/92, to be published in the Proceedings of the 27th Rencontres de Moriond (1992). 
[3] T. Banks, Phys. Lett. B272 (1991) 75.

[4] I. Montvay, Nucl. Phys. B (Proc. Suppl.) 26 (1992) 57.

[5] I-H. Lee and R.E. Shrock, Nucl. Phys. B305 (1988) 305.

[6] I. Montvay, Phys. Lett. 199B (1987) 89.

[7] L. Lin and H. Wittig, Z. Phys. C54 (1992) 331.

[8] C. Frick, T. Trappenberg, L. Lin, G. Münster, M. Plagge, I. Montvay and H. Wittig, DESY preprint 92-111.

[9] L. Lin and H. Wittig, contribution to these proceedings.

[10] M.F.L. Golterman and D.N. Petcher, Phys. Lett. 225B (1989) 159.

[11] K. Farakos, G. Koutsoumbas, L. Lin, J.P. Ma, I. Montvay and G. Münster, Nucl. Phys. B350 (1991) 474.

[12] L. Lin, I. Montvay, G. Münster and H. Wittig, Nucl. Phys. B355 (1991) 511.

[13] I-H. Lee and J. Shigemitsu, Phys. Lett. 178B (1986) 93.

[14] S. Aoki, I-H. Lee and R.E. Shrock, Phys. Lett. 178B (1986) 93.

[15] J. Kuti, Nucl. Phys. B (Proc. Suppl.) 9 (1989) 55; and references therein.

[16] M. Maggiore and M. Shifman, University of Minnesota preprint TPI-MINN-92/2-T.

[17] L.H. Karsten and J. Smit, Nucl. Phys. B183 (1981) 103.

[18] E. Seiler and I.O. Stamatescu, Phys. Rev. D25 (1982) 2177.

[19] A. Coste, C. Korthals-Altes and O. Napoly, Nucl. Phys. B289 (1987) 645;

O. Napoly, Nucl. Phys. B (Proc. Suppl.) 4 (1988) 498.

[20] W.A. Bardeen, Phys. Rev. 184 (1969) 1848.

[21] I. Montvay, to be published.

[22] D. Espriu and R. Tarrach, Z. Phys. C16 (1982) 77.

[23] J. Smit and C. Vink, Nucl. Phys. B298 (1988) 557. 